\title{
Force Sensorless Compliance Control of a Lower-limb Exoskeleton Robot
}

\section{Chung-Hsien Kuo1,*, Andika Pramanta Yudha ${ }^{2}$, and Suvendu Kumar Mohapatra ${ }^{3}$}

${ }^{1}$ Department of Electrical Engineering, National Taiwan University of Science and Technology, Taipei 106, Taiwan; and Department of Biomedical Engineering, National Defense Medical Center, Taipei 114, Taiwan

${ }^{2}$ Department of Electrical Engineering, National Taiwan University of Science and Technology, Taipei 106, Taiwan

${ }^{3}$ Industry 4.0 Center, National Taiwan University of Science and Technology, Taipei 106, Taiwan

(Received 9 August 2017; Accepted 2 October 2017; Published on line 1 March 2018)

*Corresponding author: chkuo@mail.ntust.edu.tw

DOI: 10.5875 /ausmt.v8i1.1565

Abstract: Lower limb rehabilitation machines are widely used to enhance the mobility function of elderly people and patients suffering from spinal cord injury and stroke. In this paper, a four degrees of freedom (DOF) lower body exoskeleton with a model-based compensation control framework is proposed to support hip-knee rehabilitation. The exoskeleton control movement is realized by designing a trajectory for each leg movement. A function approximation technique (FAT) based adaptive control is applied to each two DOF legs during a rehabilitation task. Using the FAT based adaptive control, the natural system dynamic is adaptively compensated for without knowing the acceleration feedback and system dynamics. Simulation results show the disturbance observer successfully estimated the user's applied leg force. The leg force is given as the input to compliance control applied in 2 DOF motors when performing a walking sequence on a treadmill. Moreover, the FAT-based adaptive control outperformed the standard proportional-differential (PD) control and could accommodate different subjects without any changes to control parameters.

Keywords: FAT Based adaptive control; exoskeleton rehabilitation; locomotors training; rehabilitation robots; disturbance observer

\section{Introduction}

Lower limb impairment can result from spinal cord injury, stroke and other diseases. Body-weight supported treadmill training (BWSTT) is generally used to treat lower limb disabilities, but BWSTT alone produces inconsistent results because rehabilitation performance is heavily reliant on the skills and experience of individual therapists.

Recent advancements in robotics and industrial automation applications can be extended to various medical applications, including the use of robots in patient rehabilitation. Lum et al. [1] proposed an approach for robot-assisted movement training and compared the effects with conventional techniques for the rehabilitation of upper-limb motor function after stroke. Similarly, Sanchez et al. [2] designed a T-WREX system to automate arm movement training following severe stroke. Khan et al. [3] proposed a robot-assisted reaching exercise which produced arm movement recovery in chronic hemiparetic stroke victims.

Numerous studies have discussed exoskeleton design for upper and lower limb patient rehabilitation. Perry et al. [4] created an upper limb exoskeleton for rehabilitation actuated using a cable transmission. Likewise, Nef et al. [5] engineered an active exoskeleton known as ARMIn III, which was modeled on the structure of the human shoulder. Krebs et al. [6] built an impedance-controlled mechanism for robot-aided rehabilitation. Tsagaralas and Caldwell [7] constructed a 2-D haptic-assisted system to minimize the pathological 
absence of motor control in patients with upper limb impairment. Rosati et al. [8] developed a wire length-controlled robot (Ne-Re-Bot) for post stroke rehabilitation. Mao and Agrawal [9] introduced a cable driven tendon system that can generate push-pull forces.

Ohnishi et al. [10] described a disturbance observer using an inverse plant model to estimate the resultant disturbance acting on the system. The robustness and tracking accuracy of industrial manipulators has been improved by applying disturbance observer (DOB) modules which are used for power assistance control. However, existing methods consider acceleration feedback. Similarly, Ugurlu et al. [11] proposed a linear DOB for an upper limb power exoskeleton, and also compensated proportional-integral-differential (PID) control for a passive assisted exoskeleton. However, this liner DOB. method uses acceleration feedback, and this exoskeleton design still relies on linear PID control with a precision manipulator model.

This paper proposes a force sensorless compliance control of a lower-limb exoskeleton robot. The major contributions of this paper are as follows:

- An adaptive control scheme for a lower limb exoskeleton is proposed to support hip-knee rehabilitation.

- The FAT-based adaptive control with acceleration-free non-linear disturbance observer position control is used to measure external torque.

- Precise manipulator system dynamic and acceleration variables are not required for the adaptive control and disturbance observer.

- The proposed exoskeleton system can achieve the desired trajectory generated by the human walking gait pattern, since the manipulator system dynamic properties and its boundedness are satisfied.

Chung-Hsien Kuo received the Ph.D. degree in mechanical engineering from National Taiwan University, Taipei, Taiwan, in 1999. Since 2012, he has been a full professor with the Department of Electrical Engineering, National Taiwan University of Science and Technology, Taipei. His current research interests include medical robotics, smart sensor and control systems, and rehabilitation engineering.

email: chkuo@mail.ntust.edu.tw

Andika Pramanta Yudha receivedthe M.S. degree in Department of Electriacl Engineering from National Taiwan University of Science and Technology, Taiwan. His current research interests include medical robotics and rehabilitation engineering.

email: andika.pramanta.yudha@gmail.com

Suvendu Kumar Mohapatra receivedthe B.Tech degree from Biju Pattnaik University, India, in June 2008, the M.Tech degree from IIIT, in June 2010 and the Ph.D. degree in Computer Science and Information Engineering from Chang Gung University, Taiwan, in July 2017. He is currently an Assistant Professor in Industry 4.0 Center of National Taiwan University of Science and Technology (Taiwan Tech), Taiwan. His current research interests include the areas of Industry 4.0, Robot automation, Cyber-physical Systems, and Big Data Analysis.

email: mohapatra.suvendu@gmail.com

\section{Lower Limb Exoskeleton Robot Control System}

Fig. 1 shows the proposed exoskeleton robot. In this approach to exoskeleton therapy, 2 degrees of freedom manipulator robot are used for each leg. The exoskeleton leg is capable of actively moving according to the modeled human-like gait trajectory and the patient leg follows the exoskeleton leg's movement. Table 1 summarizes the specifications of the proposed exoskeleton.

\begin{tabular}{ccc}
\multicolumn{4}{c}{ Table 1. EXOSKELETON $2-D O F$} & LEG MECHANICAL SPESIFICATION. \\
\hline \hline metrics & $1^{\text {st }}$ Joint & $2^{\text {nd }}$ Joint \\
\hline$m_{i}(\mathrm{~kg})$ & 4.5 & 2.0 \\
$l_{i}(\mathrm{~m})$ & 0.42 & 0.42 \\
$l_{i}\left(\frac{k g}{m^{2}}\right)$ & 0.2646 & 0.1176 \\
\hline
\end{tabular}

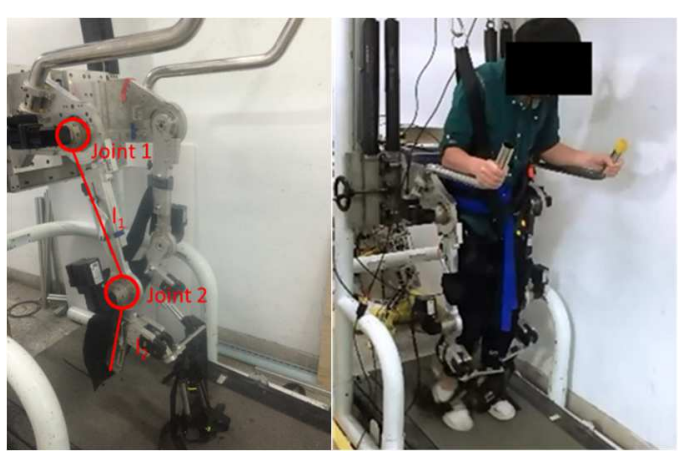

Fig. 1. 2 DOF Manipulator based Exoskeleton in the real view.

This section describes the use of position control in our exoskeleton robot and the disturbance observer as the force estimator. Later, position control, and disturbance observer are used for compliance control. Fig. 2 provides an overall diagram block.

\section{Impedance Control}

In this research, the compliance control is designed for 2-DOF exoskeleton leg. To achieve this, impedance control [12] is used as the control reference for adaptive control. Impedance control can adapt to external torque and is calculated by Eq. (1),

$$
\begin{gathered}
\ddot{q}_{m}=-M_{m}^{-1}\left(K_{m}\left(q_{m}-q_{d}\right)\right. \\
\left.+B_{m}\left(\dot{q}_{m}-\dot{q}_{d}\right)-\hat{\tau}_{d}\right)
\end{gathered}
$$

where $\ddot{q}_{m}, \dot{q}_{m}, q_{m} \in \mathfrak{R}^{2}$ and $M_{m}, B_{m}, K_{m} \in \mathfrak{R}^{2 \times 2}$ are respectively the model joint acceleration, velocity, position vector, mass constant, damping constant, and spring constant. An integration method is used to obtain joint velocity and position.

\section{FAT Based Adaptive Control}

In the FAT-based adaptive control, regressor-free ausMT Vol. 8 No. 1 (2018) 
adaptive control is used for the motor position control [13]. Specifically, this control does not require acceleration feedback or overall system dynamic. Therefore, the manipulator system dynamic is changed as shown in Eq. (2), where the $M(q) \in W^{n \times n}$ is the inertia matrix, $C(q, \dot{q}) \in \Re^{n \times n}$ is the Coriolis and centrifugal term, $g(q) \in \Re^{n}$ is gravity, $\ddot{q}$ is the joint rotational acceleration and $\psi(t)$ is the unknown time varying function.

$$
\psi(t)=M(q) \ddot{q}+C(q, \dot{q}) \dot{q}+g(q)-\ddot{q}
$$

The torque input for the manipulator is shown in Eq. (3), where $\widehat{\Psi} \in \mathcal{R}^{n}$ is the estimate of $\psi, K_{p} \in \mathcal{R}^{n \times n}$ is the proportional gain, the $K_{d} \in \Re^{n \times n}$ is the derivative gain, and $e=\left(q-q_{d}\right), \dot{e}=\left(\dot{q}-\dot{q}_{d}\right) \in \mathfrak{R}^{n}$ are respectively the rotational position and rotational velocity errors.

$$
\tau=\hat{\psi}+\ddot{q}_{m}-K_{d} \dot{e}-K_{p} e
$$

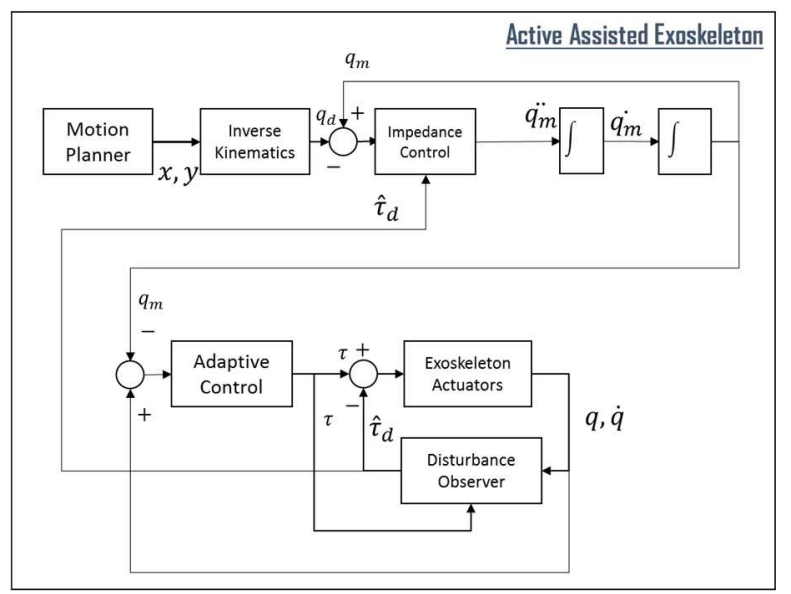

Fig. 2. Exoskeleton Control Scheme.

The desired rotational positions are obtained for first and second joints based on inverse kinematic output as described in the previous section. Then the closed loop system dynamic is shown in Eq. (4).

$$
\ddot{e}+K_{d} \dot{e}+K_{p} e=\hat{\psi}-\psi
$$

The update law is designed for $\psi \hat{\imath} \rightarrow \psi$, and the tracking $e$ is converged asymptotically. To design the update law, FAT is applied to represent $\psi$ and $\psi$ as shown in Eqs. (5) and (6).

$$
\begin{gathered}
\psi(t)=W^{T} z(t)+\varepsilon \\
\hat{\psi}=\hat{W}^{T} z+\varepsilon
\end{gathered}
$$

Based on Eqs. (5) and (6), $W \in \mathcal{R}^{\beta n \times n}$ is the weighting matrix, $z(t) \in \mathcal{R}^{\beta n \times 1}$ is the basis function vector, and $\varepsilon$ is the approximation error. Hence, the state space is formed by using Eqs. (4), (5), and (6), as shown in Eq. (7).

$$
\dot{x}=A x-B\left(\tilde{W}^{T} z+\varepsilon\right)
$$

In Eq. (7),

$$
A=\left[\begin{array}{cc}
0 & I_{n} \\
-K_{p} & -K_{D}
\end{array}\right] B=\left[\begin{array}{l}
0 \\
I_{n}
\end{array}\right] \quad x=\left[\begin{array}{l}
e \\
\dot{e}
\end{array}\right]
$$

To ensure stability, the Lyapunov-like function candidate is considered as shown in Eq. (8).

$$
V=\frac{1}{2} x^{T} P x+\operatorname{Tr}\left(W^{T} \Gamma_{1} \tilde{W}\right)
$$

In Eq. (8), $\Gamma_{1}=\Gamma_{1}^{T} \in \mathfrak{R}^{\beta n \times \beta n}$ is a positive definite matrix and $P=P^{T} \in \mathfrak{R}^{\beta n \times \beta n}$ is a positive definite matrix satisfying the Lyapunov equation $A^{T} P+P A=-Q$ for a given positive definite matrix $Q \in \mathfrak{R}^{2 n \times 2 n}$. The time derivative of $\mathrm{V}$ along trajectory of Eq. (8) is shown in Eq. (9).

$$
\dot{V}=\frac{1}{2} x^{T} Q x-\operatorname{Tr}\left(W^{T} z x^{T} P B+W^{T} \Gamma_{1} \dot{\hat{W}}\right)
$$

Therefore, based on Eq. (9), the update law with $\sigma$ modification is designed as shown in Eq. (10), where $\sigma$ is a positive number.

$$
\dot{\hat{W}}=-\Gamma_{1}^{-1}\left(z x^{T} P B+\sigma \hat{W}\right)
$$

The approximation error $\varepsilon$ is ignored if a sufficient number of basis functions are used. Otherwise, if there is no $\sigma$-modification, then we have $\dot{V}=-\frac{1}{2} x^{T} Q x \leq 0$.

\section{Disturbance Observer}

External torque is considered as an external disturbance for a manipulator based robot. In this research, a non-linear disturbance observer is used to determine the external torque as a disturbance without using a torque sensor.

Manipulator system dynamics include joint acceleration, velocity, and position as shown in Eq. (11). It is very hard to design a disturbance observer since acceleration sensor is noisy. Therefore, in this subsection, the non-linear disturbance observer [14] is used with free acceleration. Some parameter settings must be determined to achieve the desired disturbance observer response or obtain any external force around the robotic environment.

Consider an $n$-DOF rigid serial manipulator system dynamic with external torque and friction force as shown in Eq. (11) below.

$$
M(q) \ddot{q}+C(q, \dot{q}) \dot{q}+g(q)+F(\dot{q})=\tau+\tau_{e x t}
$$

where, $\hat{M}(q), \hat{C}(q)$, and $\hat{g}(q)$ are respectively the estimated values of Inertia matrix, Coriolis, and gravity terms. In addition, $\Delta \mathrm{M}, \Delta \mathrm{C}$, and $\Delta \mathrm{g}$ are additive uncertainties present in robot model as respectively shown in Eqs. (12), (13), and (14).

$$
\begin{gathered}
M(q)=\hat{M}(q)+\Delta M \\
C(q, \dot{q})=\hat{C}(q, \dot{q})+\Delta C
\end{gathered}
$$




$$
g(q)=\hat{g}(q)+\Delta g
$$

Then, the lumped disturbance vector $\tau_{d}$ with all dynamic uncertainties, joint friction and external torque are defined in Eq. (15).

$$
\tau_{d}=\tau_{\text {ext }}-\Delta M \ddot{q}-\Delta C \dot{q}-\Delta g-F(\dot{q})
$$

Hence, the estimated values of manipulator system dynamic are changed to Eq. (16).

$$
\hat{M}(q) \ddot{q}+\hat{C}(q, \dot{q}) \dot{q}+\hat{g}(q)=\tau+\tau_{d}
$$

Based on Eq. (16), the basic disturbance observer is shown in Eq. (17) below.

$$
\dot{\hat{\tau}}_{d}=-L \hat{\tau}_{d}+L\{\hat{M}(q) \ddot{q}+\hat{C}(q, \dot{q})+\hat{g}(q)-\tau\}
$$

Based on Eq. (17), $L$ is the observer gain matrix. By defining $\Delta \tau_{d}=\tau_{d}-\hat{\tau}_{d}$, the torque disturbance error dynamic is presented in Eq. (18).

$$
\dot{\hat{\tau}}_{d}=L \Delta \tau_{d}, \Delta \dot{\tau}_{d}=\dot{\tau}_{d}-L \Delta \tau_{d}
$$

The disturbance observer requires acceleration feedback to obtain the disturbance torque as shown in Eq. (17). However, the disturbance observer proposed by Mohammadi et al. [14] has the same error dynamic even without acceleration feedback. We propose an acceleration-free disturbance observer. First, we define the auxiliary $z$ as in Eq. (19)

$$
z=\hat{\tau}_{d}-p(q, \dot{q})
$$

Vector $p(q, \dot{q})$ is defined by using observer gain matrix as mention previously, and is shown in Eq. (20).

$$
\frac{d}{d t} p(q, \dot{q})=L(q) \hat{M}(q) \ddot{q}
$$

Using Eqs. (16), (17) and (19) and taking derivative of Eq. (20), the modified disturbance observer is shown in Eq. (21) [14].

$$
\begin{aligned}
\dot{z} & =-L z+L\{\hat{C}(q, \dot{q}) \dot{q}+\hat{g}(q)-\tau-p(q, \dot{q})\} \\
\hat{\tau}_{d} & =z+p(q, \dot{q})
\end{aligned}
$$

From Eq. (21), the modified disturbance observer error dynamic is the same as the basic disturbance observer, as proven in Eq. (22).

$$
\begin{aligned}
\Delta \dot{\tau}_{d}= & \dot{\tau}_{d}-\dot{\hat{\tau}}_{d}=\dot{\tau}_{d}-\dot{z}-\frac{d}{d t} p(q, \dot{q}) \\
= & \dot{\tau}_{d}+L(q) z-L(q)\{c(q, \dot{q}) \dot{q} \\
& +g(q)-\tau-P(q, \dot{q})\} \\
& -L(q) \hat{M}(q) \ddot{q} \\
& =\dot{\tau}_{d}+L(q)\left\{\hat{\tau}_{d}-p(q, \dot{q})\right\}-L(q) \\
& \left\{-\hat{M}(q) \ddot{q}+\tau_{d}-P(q, \dot{q})\right\} \\
& -L(q) \hat{M}(q) \ddot{q} \\
= & \dot{\tau}_{d}-L(q)\left(\tau_{d}-\hat{\tau}_{d}\right) \\
= & \dot{\tau}_{d}-L(q) \Delta \tau_{d}
\end{aligned}
$$

The gain matrix $P$, and $L$ play an important role for designing the disturbance observer gain. The proposed matrix is shown in Eq. (23).

$$
L(q)=X^{-1} \hat{M}^{-1}(q)
$$

In Eq. (23), matrix $L(q)$ is invertible, and has the property of the disturbance observer. The estimated inertia matrix is a positive definite matrix and is thus invertible. From Eq. (20) the matrix is defined in Eq. (24) as:

$$
P(q, \dot{q})=X^{-1} \dot{q}
$$

Finally, using the LMI method proposed in [14], the invertible gain matrix $X^{-1}$ can be found in Eq. (25).

$$
X^{-1}=\frac{1}{2}\left(\zeta+2 \beta \sigma_{2}\right) I_{2 \times 2}
$$

In Eq. (25), $\zeta$ is $\|\dot{\hat{M}}(q)\|$, the $\sigma_{2}$ is $\|\hat{M}(q)\|$, and the $\beta$ is the desired convergence rate.

\section{Human Gait Pattern}

In this study, the human gait pattern is defined as the motion planner for our exoskeleton trajectory. Fig. 3 illustrates the human walking pattern.

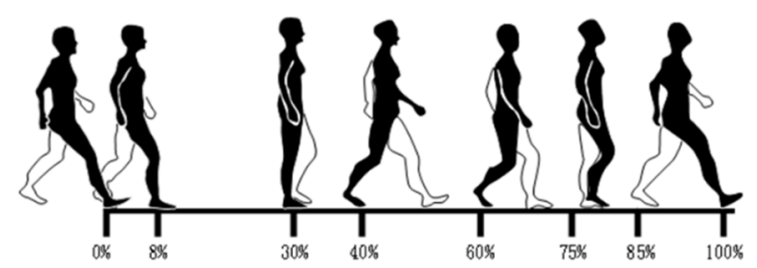

Fig. 3. Human walking pattern.

Fig. 4 shows the trajectory gait for hip and knee joints for a 50 cycle/step walk.

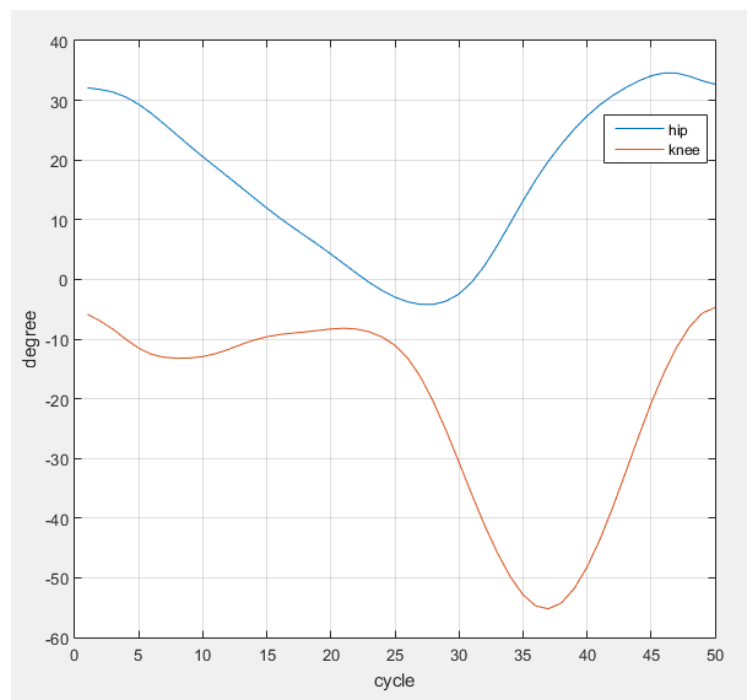

Fig. 4. Generated hip and knee joint trajectory for 50 cycle/step walk. 
Since the inverse kinematic is used to obtained the desired hip and knee joint position, the end effector trajectory is plotted in a 2D Cartesian diagram as the input for the inverse kinematic. Using the MATLAB curve fitting tool, the end effector trajectory in the $2 \mathrm{~d}$ Cartesian diagram is shown in Eq. (26).

$$
\begin{aligned}
P_{x}= & a_{x 0}+a_{x 1} \cos \left(t w_{x}\right)+b_{x 1} \sin \left(t w_{x}\right) \\
& +a_{x 2} \cos \left(2 t w_{x}\right)+b_{x 2} \sin \left(2 t w_{x}\right) \\
& +a_{x 3} \cos \left(3 t w_{x}\right)+b_{x 3} \sin \left(3 t w_{x}\right) \\
P_{z} & =a_{z 0}+a_{z 1} \cos \left(t w_{z}\right)+b_{z 1} \sin \left(t w_{z}\right) \\
& +a_{z 2} \cos \left(2 t w_{z}\right)+b_{z 2} \sin \left(2 t w_{z}\right) \\
& +a_{z 3} \cos \left(3 t w_{z}\right)+b_{z 3} \sin \left(3 t w_{z}\right) \\
& +a_{z 4} \cos \left(4 t w_{z}\right)+b_{z 4} \sin \left(4 t w_{z}\right) \\
& +a_{z 5} \cos \left(5 t w_{z}\right)+b_{z 5} \sin \left(5 t w_{z}\right)
\end{aligned}
$$

For our case, the parameters in Eq. (26) are chosen as $a_{x 0}=5.422, a_{x 1}=25.82, b_{x 1}=12.61, a_{x 2}=6.249, b_{x 2}=-6.304$,

$a_{x 3}=0.8893, b_{x 3}=-0.8885, w_{x}=0.1256, a_{z 0}=-77.98$,

$a_{z 1}=2.084, b_{z 1}=-4.941, a_{z 2}=-0.9565, b_{z 2}=2.42$,

$a_{z 3}=1.993, b_{z 3}=0.7234, a_{z 4}=0.5084, b_{z 4}=-0.6855$,

$a_{z 5}=-0.005, b_{z 2}=2.42, a_{z 3}=1.993, b_{z 3}=0.7234, a_{z 4}=0.5084$,

$b_{z 4}=-0.6855, a_{z 5}=-0.005, b_{z 5}=-0.1436, w_{z}=0.1257$

The end effector based on Eq. (26) in 2D Cartesian space is shown in Fig. 5.

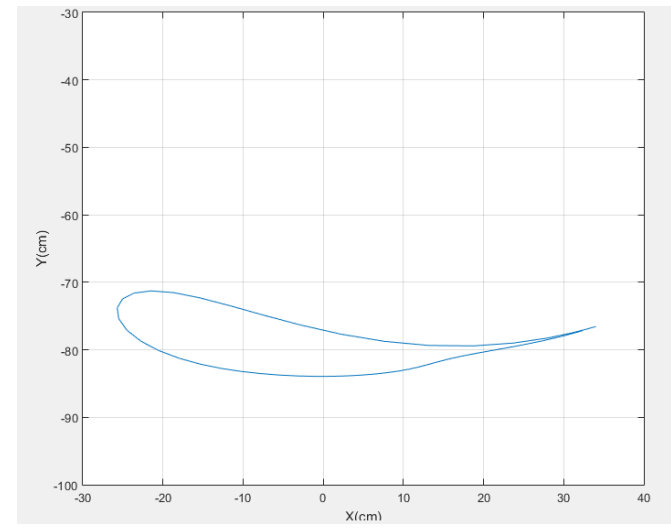

Fig. 5. End effector trajectory based on human gait.

\section{Experiments and Results}

\section{Simulation Results}

Simulations and real-world experiments were performed to assess system performance. The simulation modeled the exoskeleton gait movement using position control to provide gait movement. The simulation results present the modeled force and the estimated force using the disturbance observer. The desired trajectory would reach and collide with the treadmill belt on the base frame. Moreover, a ground contact force exists between the treadmill and patient's foot. Hence, the disturbance observer is used to estimate all external forces affecting all joints for compliance control. Simulations were performed using ODE45 in MATLAB.

The simulations were run for 50 seconds using the following adaptive control parameters: $K_{p}, K_{d}, P$ gain $\Gamma_{1}$, and $\sigma$-modification is

$$
\begin{aligned}
K_{p} & =\left[\begin{array}{cc}
800 & 0 \\
0 & 600
\end{array}\right], \\
K_{d} & =\left[\begin{array}{cc}
200 & 0 \\
0 & 120
\end{array}\right], \\
P & =\left[\begin{array}{cccc}
10^{4} & 0 & 2 \times 10^{5} & 0 \\
0 & 2 \times 10^{4} & 0 & 4 \times 10^{5} \\
2 \times 10^{5} & 0 & 10^{4} & 0 \\
0 & 4 \times 10^{5} & 0 & 2 \times 10^{4}
\end{array}\right],
\end{aligned}
$$

$\Gamma_{1}=1000 \mathrm{I}_{32}$, and $\sigma=1$. The initial weighting value $\hat{W}(0) \in \mathfrak{R}^{32 \times 2}$ is zero.

The disturbance observer parameters are set at $\zeta=\|\dot{\hat{M}}(q)\|=0.4591, \sigma_{2}=\|M(q)\|=0.3528$ and $\beta=10 \quad$, so $X^{-1}=\left[\begin{array}{cc}3.7576 & 0 \\ 0 & 3.7576\end{array}\right]$. The approximation of inertia matrix $\widehat{M}_{(q)}$ as the gain in $L$ needs to be as small as possible to increase the disturbance observer convergence rate. In this simulation, since $\widehat{M}_{(q)}$ must be a positive, definite and symmetric matrix, it is set at $\hat{M}(q)=0.01 M(q)$,

$$
\hat{M}(q)=\left[\begin{array}{cc}
0.003528 \cos \left(q_{2}\right)+0.0102 & 0.001764 \cos \left(q_{2}\right)+0.002058 \\
0.001764 \cos \left(q_{2}\right)+0.002058 & 0.002058
\end{array}\right]
$$

The estimated Coriolis and gravity terms to be used in the disturbance observer are,

$$
\begin{aligned}
& \hat{C}(q, \dot{q})=\left[\begin{array}{cc}
-0.1764 \dot{q}_{2} \sin \left(q_{2}\right) & -0.1764 \sin \left(q_{2}\right)\left(\dot{q}_{1}+\dot{q}_{2}\right) \\
0.1764 \dot{q}_{1} \sin \left(q_{2}\right) & 0
\end{array}\right] \\
& \hat{G}(q)=\left[\begin{array}{c}
4.116 \cos \left(q_{1}\right)+17.493 \cos \left(q_{1}+q_{2}\right) \\
4.116 \cos \left(q_{1}+q_{2}\right)
\end{array}\right]
\end{aligned}
$$

The position control performance tracking is shown in Fig. 6. Based on Fig. 6, the controller can track the desired position well. This results in an error in the motor position since the end effector leg touches the ground, thus the exoskeleton leg can adapt to it. Fig. 7 compares the estimated force (blue) and modeled force (red) in the $y$ direction. The modeled force is tracked sufficiently through performance tracking. Torque error occurs occasionally but it converges again to the modeled force. Since we are using adaptive control for position control, the estimated parameter $\hat{\psi}$ is shown in Fig. 8. Initially, each estimated parameter has a 
significant overshoot so that the controlled motor position is converged to the desired position as quickly as possible.

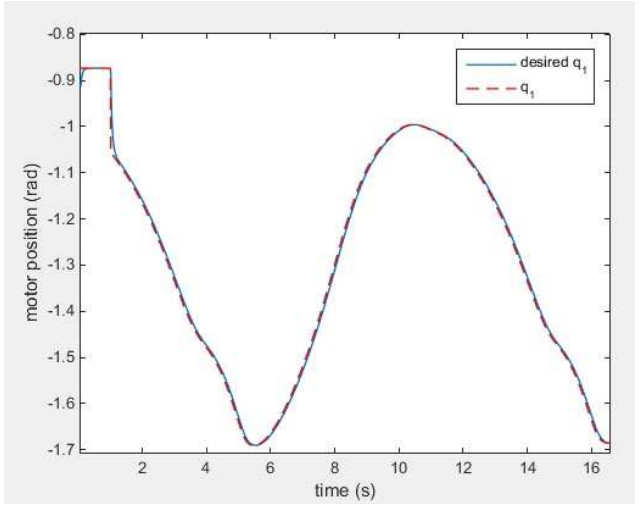

(a)

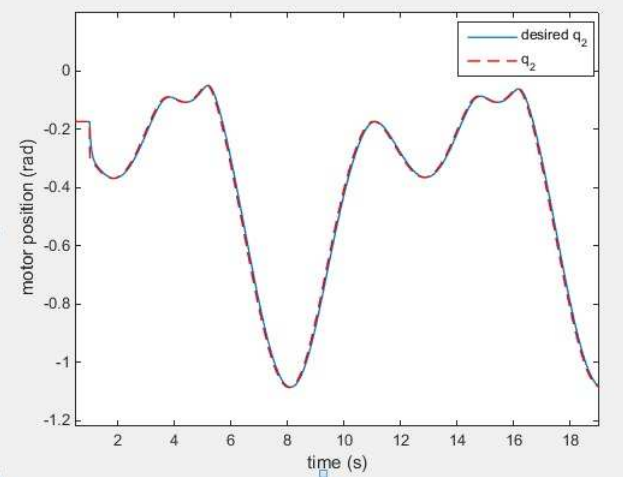

(b)

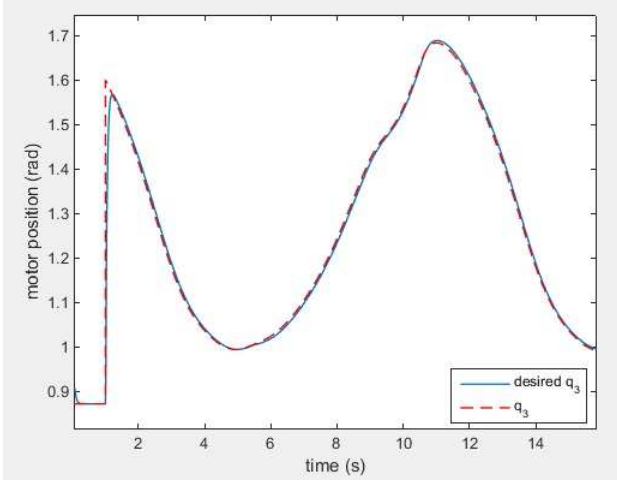

(c)

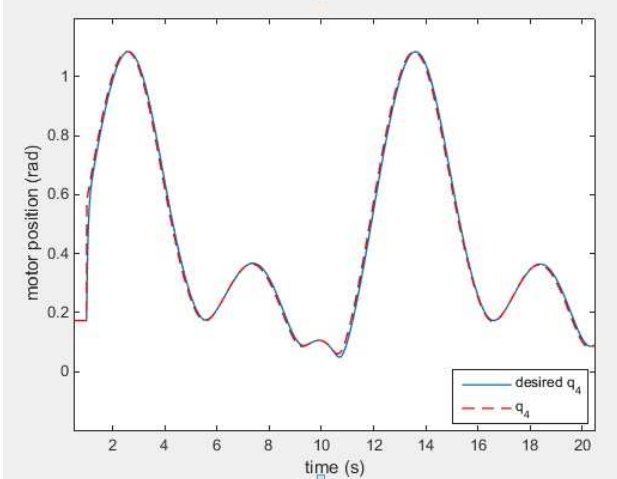

(d)

Fig. 6. Motor position performance tracking of (a) $1^{\text {st }}$ joint, (b) $2^{\text {nd }}$ joint, (c) $3^{\text {rd }}$ joint, and (d) $4^{\text {th }}$ joint.

\section{Exoskeleton Robot Experiment Results}

Experiments were performed using a $\mathrm{PC}$ with an i5 $2.53 \mathrm{GHz}$ dual core processor, Microsoft Visual C\# as the software development environment and language programming, and smart motors as the actuators. A serial RS232 is used for communication between the PC and all motor actuators. The communication data package is designed according to the Smart Motor communication protocol.

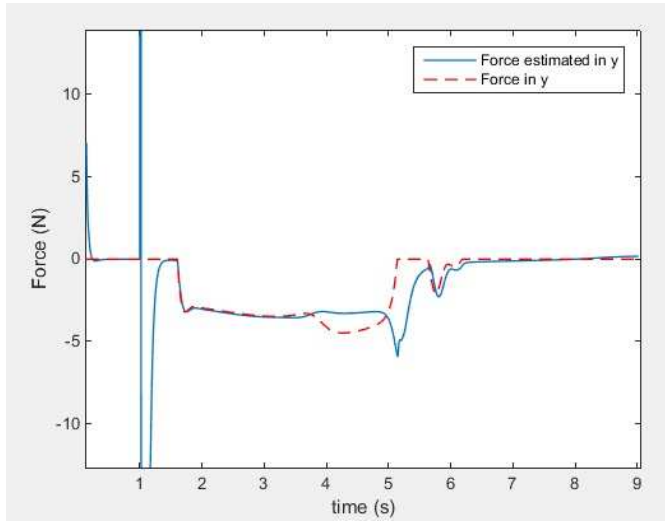

(a)

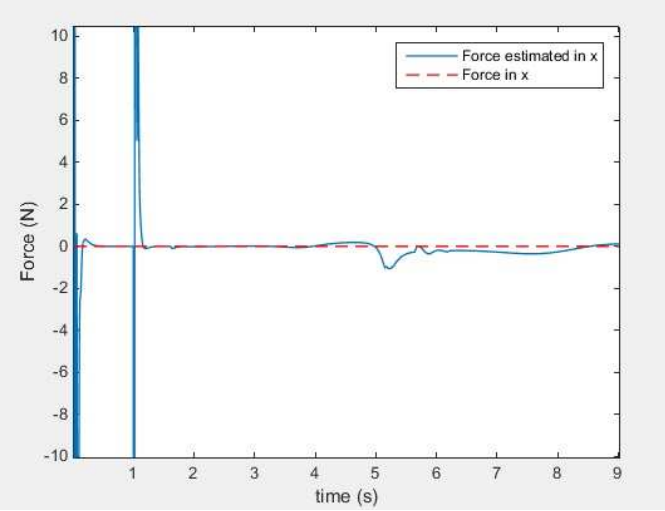

(b)

Fig. 7. Estimated and modeled force in (a) $y$ direction and (b) $x$ direction.

In this experiment, joint position control is performed with compliance capability to exert the compliant force interaction with the subject and treadmill belt platform. The FAT-based parameters are the same as those used in the simulation, but the disturbance observer convergence rate is changed to $\beta=1$ as the noisy torque disturbance should not be an input of impedance control and FAT-based adaptive control. The impedance parameters are chosen as,

$$
K_{m}=\left[\begin{array}{cc}
600 & 0 \\
0 & 600
\end{array}\right], B_{m}=\left[\begin{array}{cc}
40 & 0 \\
0 & 40
\end{array}\right], M_{m}=\left[\begin{array}{cc}
0.5 & 0 \\
0 & 0.5
\end{array}\right]
$$

The output positions in Fig. 9 show the real motor 1 position can track the model position well, especially 


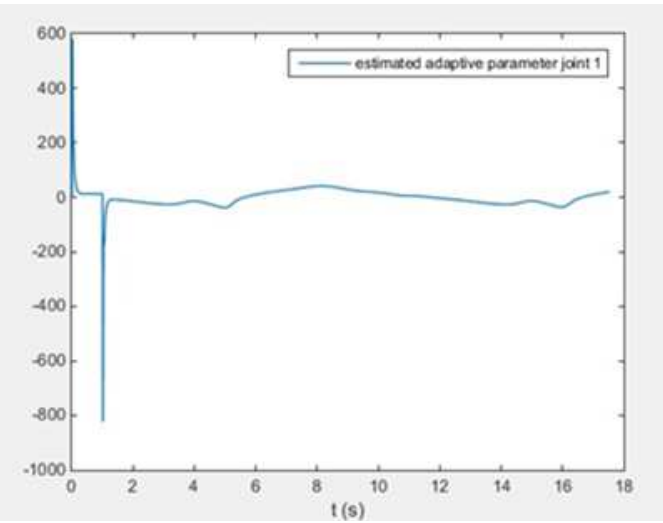

(a)

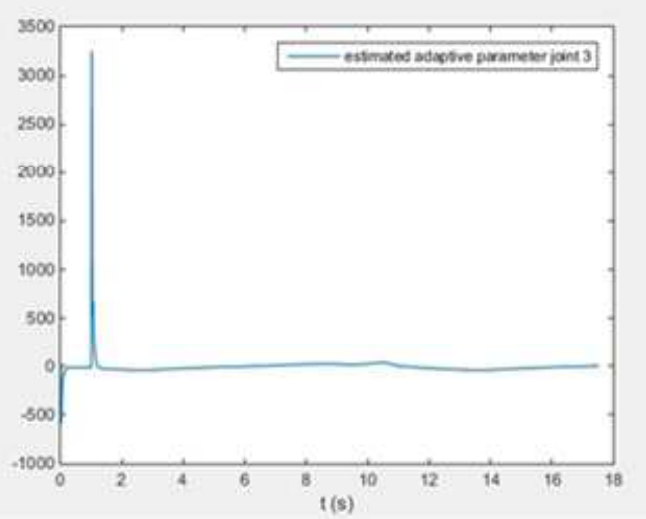

(c)

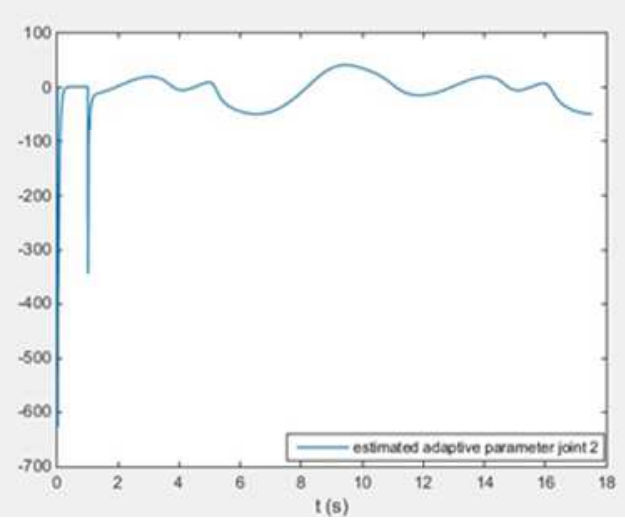

(b)

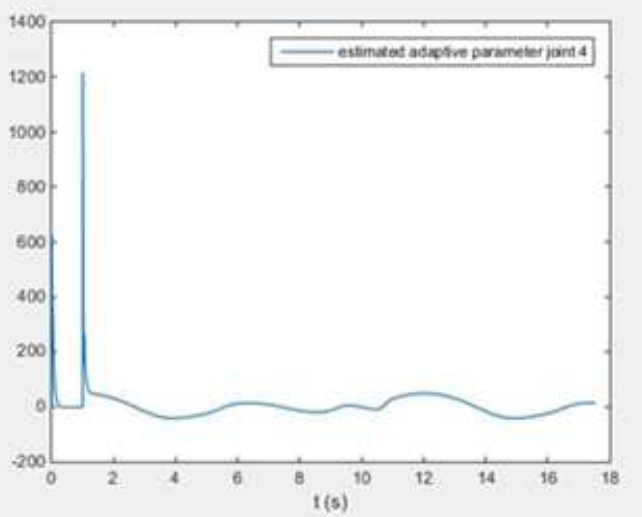

(d)

Fig. 8. Estimated parameters of (a) $1^{\text {st }}$ joint (b) $2^{\text {nd }}$ joint (c) $3^{\text {rd }}$ joint (d) $4^{\text {th }}$ joint.

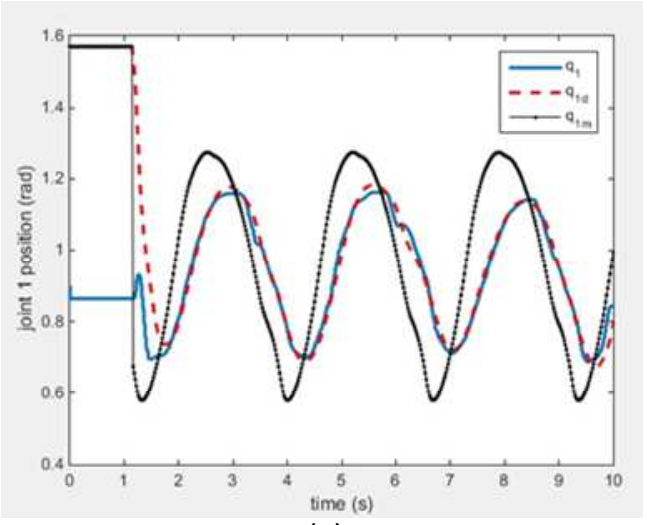

(a)

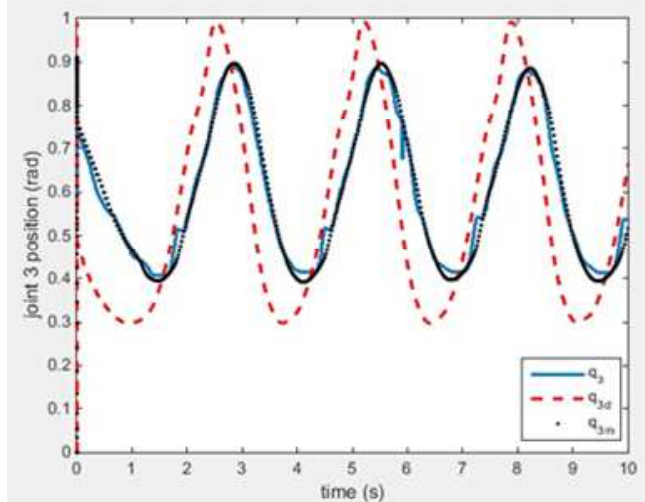

(c)

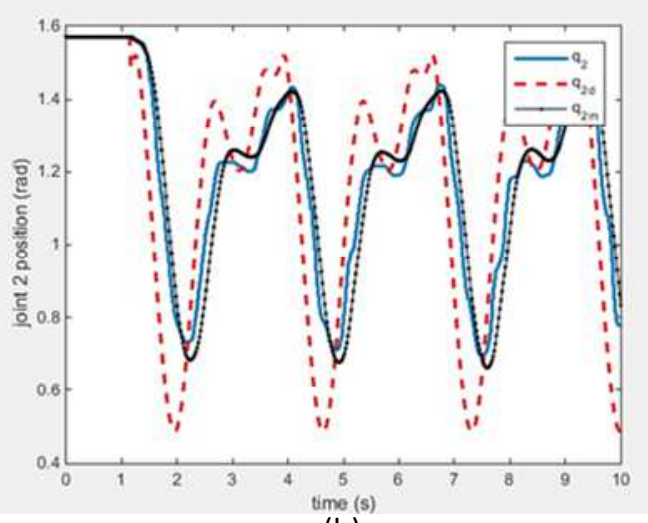

(b)

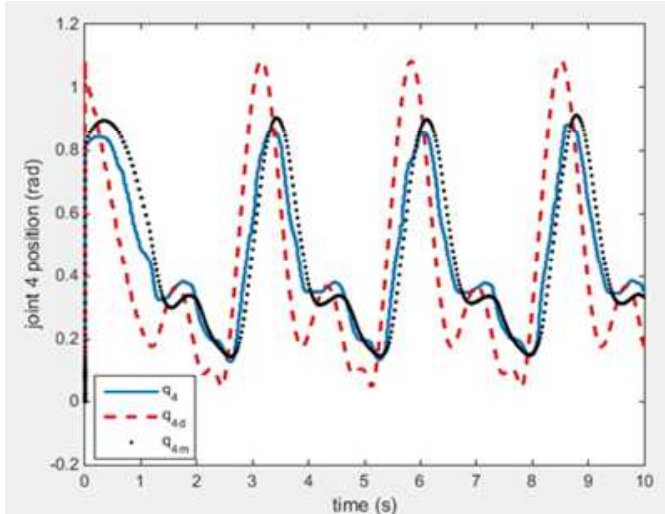

(d)

Fig. 9. Experimental result performance tracking for (a) $1^{\text {st }}$ joint, (b) $2^{\text {nd }}$ Joint, (c) $3^{\text {rd }}$ joint, (d) $4^{\text {th }}$ joint. 
for the upper joints for legs 1 and 2. There is a big error on the trajectory tracking, especially in the impedance control as the exoskeleton system still detects the gravity as the external torque. The disturbance observer outputs for all joints are shown in Fig. 10. The graphic performance tracking shows the disturbance observer outputs fluctuate, and determining the source of the problem requires comparing the torque graphic outputs in Fig. 11.

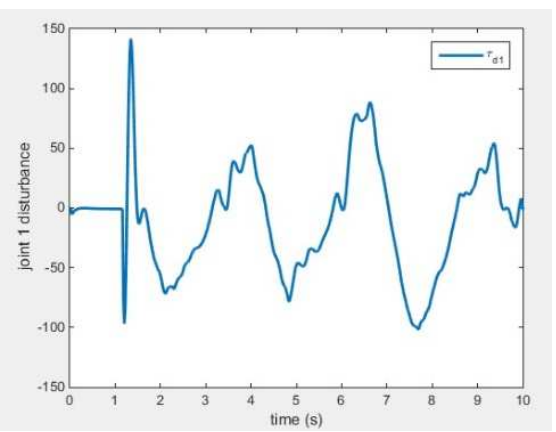

(a)

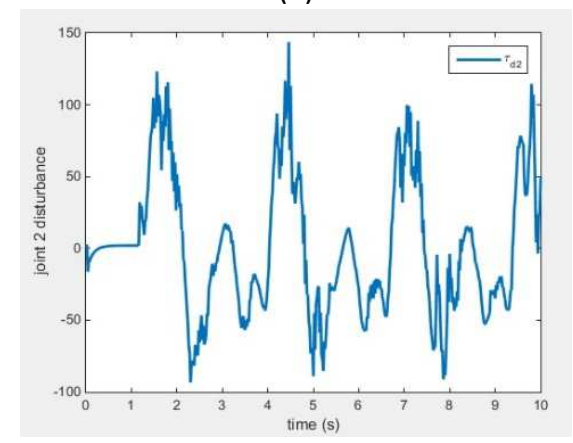

(b)

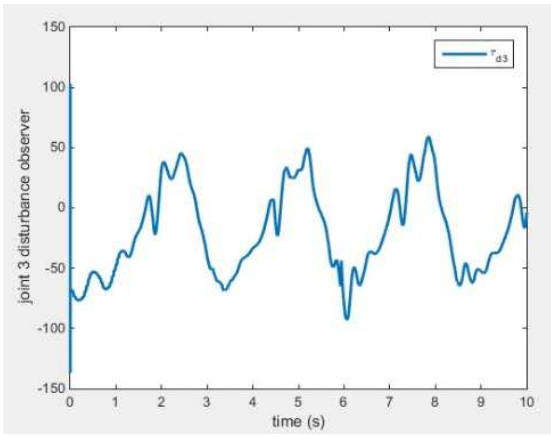

(c)

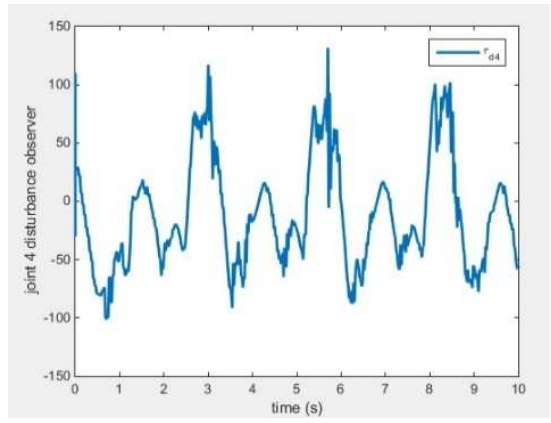

(d)

Fig. 10 (a) $1^{\text {st }}$ Joint, (b) $2^{\text {nd }}$ Joint, (c) $3^{\text {rd }}$ Joint and (d) $4^{\text {th }}$ Joint disturbance observer output.

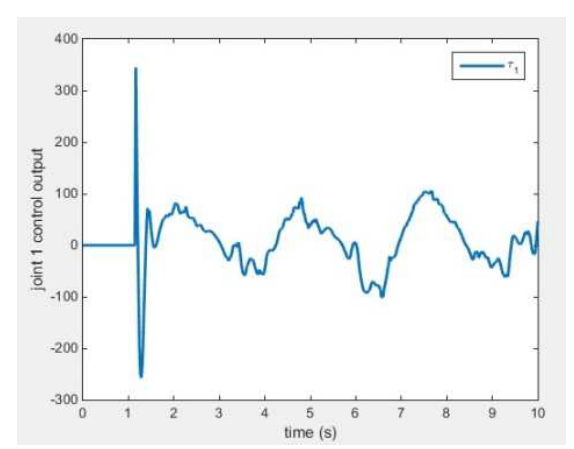

(a)

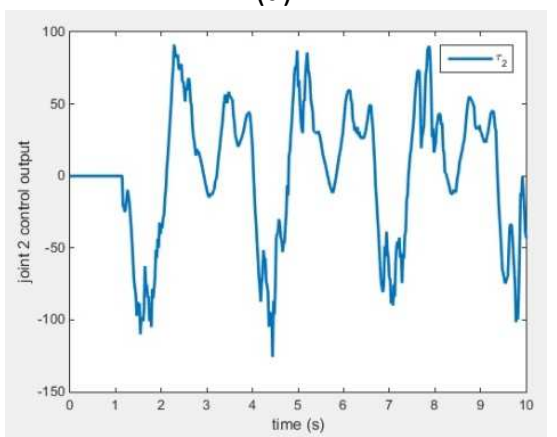

(b)

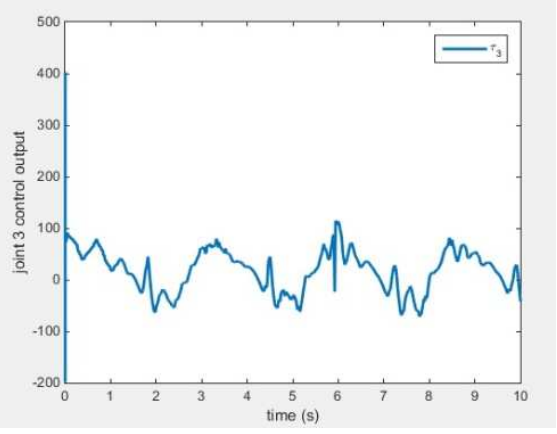

(c)

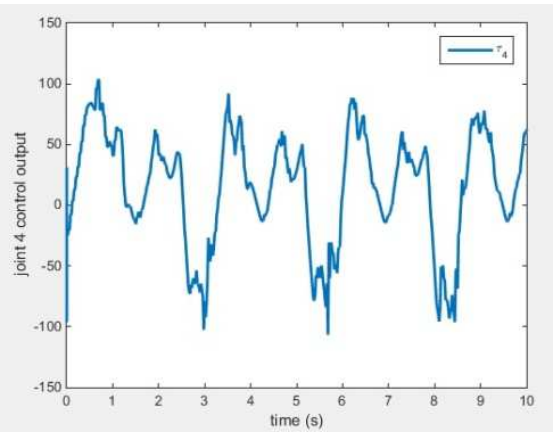

(d)

Fig. 11. (a) $1^{\text {st }}$ joint, (b) $2^{\text {nd }}$ Joint, (c) $3^{\text {rd } J o i n t, ~ a n d ~(d) ~} 4^{\text {th }}$ Joint torque output.

Comparing the disturbance observer and torque graphics, we find that the disturbance observer output is actually in the opposite direction of the torque output. As seen in the disturbance observer equation (21), this problem is due to differences between the gravity and 
Coriolis manipulator matrices in the real system. Thus, these matrix variables can't compensate for the motor torque in the absence of external torque.

$$
\tau=M(q) \ddot{q}+C(q, \dot{q}) \dot{q}+g(q) .
$$

The exoskeleton trajectory changes when force is exerted by the leg, as shown in the disturbance graph. The amplitude initially fluctuates but then returns to normal. The compliance effect can be seen by observing the end effector position as shown in Fig. 12 and Fig. 13. Note that, more than 1 step is performed in 10 seconds, hence more than 1 blue line is shown in the graphic. Application of external torque changes the trajectory. The overall rehabilitation and training of continuous action is shown in Fig. 14.

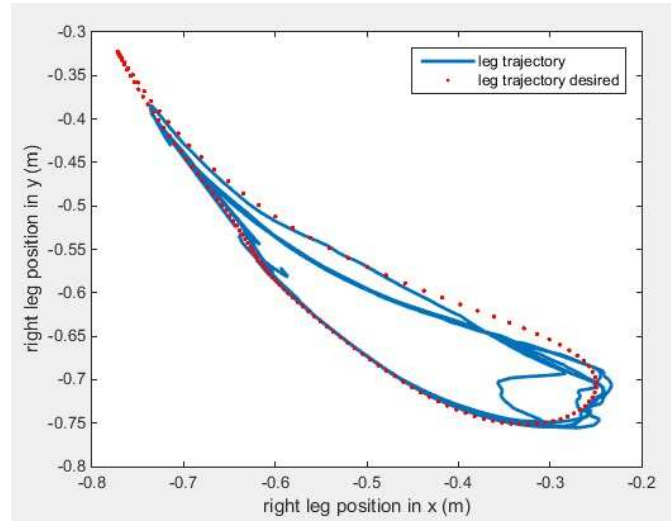

Fig. 12. Right leg trajectory.

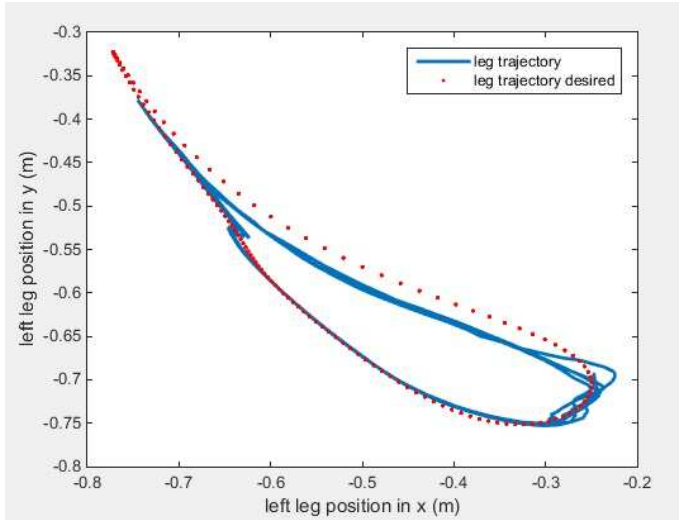

Fig. 13. Left leg trajectory

\section{Conclusion and Future Work}

This research describes the development of an exoskeleton rehabilitation system based on position control with compliance capacity. Simulation results clearly show that the force sensorless disturbance observer performs very well in estimating the modeled external force affecting the exoskeleton system while walking, thus the joint can adapt to environmental changes. Simulation results also prove that the FAT-based adaptive control can be used effectively as a position control without acceleration feedback or system dynamics in the control system.

In the real-world experiment, the disturbance observer performs differently, showing the opposite value for the control torque output since the system dynamic used for the disturbance observer model cannot compensate for real torque to reach the desired position. But the function approximation technique (FAT) based adaptive control performs well in tracking the model reference control even without acceleration feedback and the awareness of the system dynamics.

Future work will use a function approximation technique (online or offline) to obtain the uncompensated system dynamic which is considered as the external torque for our disturbance system, thus improving disturbance observer output, and moving the proposed system architecture towards real-world implementation.

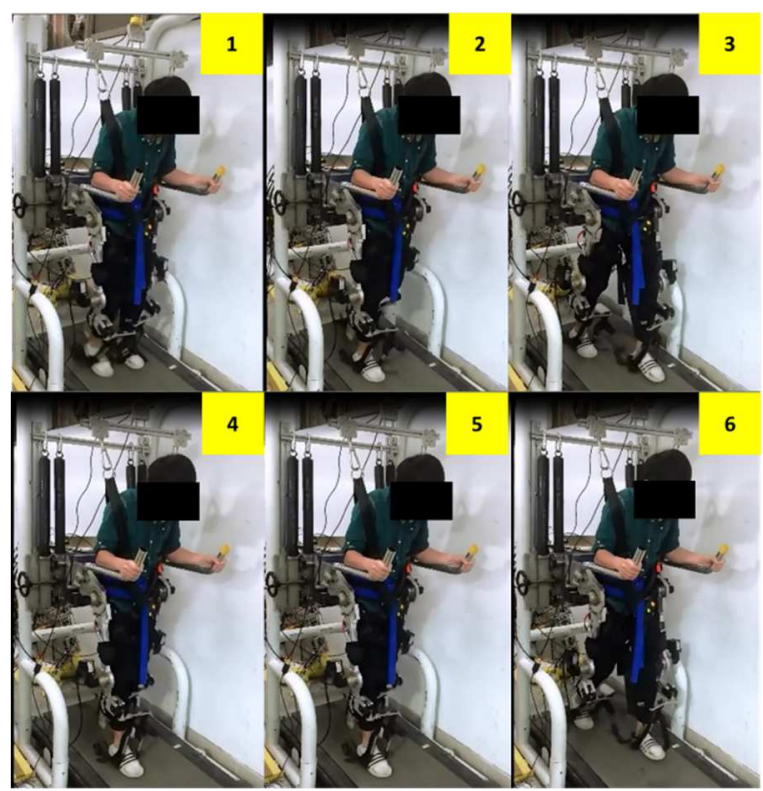

Fig. 14. Rehabilitation and training of continuous action view.

\section{References}

[1] P. S. Lum, C. G. Burgar, P. C. Shor, M. Majmundar, and M. Van der Loos, "Robot-assisted movement training compared with conventional therapy techniques for the rehabilitation of upper limb motor function following stroke," Archives Phys. Med. Rehabil., vol. 83, no. 7, pp. 952-959, Jul. 2002. doi: 10.1053/apmr.2001.33101

[2] R. J. Sanchez, J. Liu, S. Rao, P. Shah, R. Smith, T. Rahman, S. C. Cramer, J. E. Bobrow, and D. J. Reinkensmeyer, "Automating arm movement training following severe stroke: Functional exercises with quantitative feedback in a gravity-reduced environment," IEEE Trans. Neural Syst. Rehabil. Eng., 
vol. 14, no. 3, pp. 378-389, Sep. 2006. doi: $10.1109 / T N S R E .2006 .881553$

[3] L. E. Kahn, M. L. Zygman, W. Z. Rymer, and D. J. Reinkensmeyer, "Robot-assisted reaching exercise promotes arm movement recovery in chronic hemiparetic stroke: A randomized controlled pilot study," J. NeuroEng. Rehabil., vol. 3, no. 12, pp. 1-13, Jun. 2006.

doi: $10.1186 \% 2 \mathrm{~F} 1743-0003-3-12$

[4] J. C. Perry, J. Rosen, and S. Burns, "Upper-limb powered exoskeleton design," IEEE Trans. Mechatronics, vol. 12, no. 4, pp. 408-417, Aug. 2007. doi: 10.1109/TMECH.2007.901934

[5] T. Nef, M. Guidali, and R. Riener, "ARMin III-Arm therapy exoskeleton with an ergonomic shoulder actuation," Appl. Bionics Biomech., vol. 6, no. 2, pp. 127-142, Jul. 2009. doi: $10.1080 / 11762320902840179$

[6] H. I. Krebs, N. Hogan, M. L. Aisen, and B. T. Volpe, "Robot-aided neuro rehabilitation," IEEE Trans. Rehabil. Eng., vol. 6, no. 1, pp. 75-87, Mar. 1998. doi: $10.1109 / 86.662623$

[7] N. G. Tsagarakis and D. G. Caldwell, "Improving mouse-based computer interaction in users with weak upper limb motion control during using a haptic assistive system," IEEE Trans. Human-Mach. System., vol. 43, no. 2, pp. 177-187, Mar. 2013. doi: $10.1109 / T S M C C .2012 .2204872$

[8] G. Rosati, P. Gallina, and S. Masiero, “Design, implementation and clinical tests of a wire-based robot for neuro rehabilitation," IEEE Trans. Neural Syst. Rehabil. Eng., vol. 15, no. 4, pp. 560-569, Dec.
2007.

doi: 10.1109/TNSRE.2007.908560

[9] Y. Mao and S. K. Agrawal, "Design of a cable-driven arm exoskeleton (CAREX) for neural rehabilitation," IEEE Trans. Robot., vol. 28, no. 4, pp. 922-931, Dec. 2007. doi: $10.1109 /$ TRO.2012.2189496

[10] K. Ohnishi, M. Shibata, and T. Murakami, "Motion control for advanced mechatronics," IEEE Trans. Mechatron., vol. 1, no. 1, pp. 56-67, Mar. 1996. doi: $10.1109 / 3516.491410$

[11] B. Ugurlu, M. Nishimura, K. Hyodo, M. Kawanishi, and T. Narikiyo, "Proof of Concept for Robot-Aided Upper Limb Rehabilitation Using Disturbance Observers," IEEE Trans. Human-Mach. System., vol. 45, no. 1, pp. 110-118, Feb. 2015 doi: $10.1109 / T H M S .2014 .2362816$

[12] C.-Y. Kai and A.-C. Huang, "A regressor-free adaptive impedance controller for robot manipulators without Slotine and Li's modification: theory and experiments," Robotica, vol. 33, no. 3, pp. 638-648, March 2015. doi: $10.1017 / \mathrm{S} 0263574714000277$

[13] C.-Y. Kai and A.-C. Huang, "A regressor-free adaptive controller for robot manipulators without Slotine and Li's modification," Robotica, vol. 31, no. 7, pp. 1051-1058, Oct. 2013.

[14] A. Mohammadi, M. Tavakoli, H. J. Marquez, and F. Hashemzadeh, "Nonlinear disturbance observer design for robotic manipulators," Control Eng. Practice, vol. 21, no.3, pp. 253-267, Mar. 2013. doi: 10.1016/i.conengprac.2012.10.008 